\title{
LncRNA MALAT1 Promotes Cancer Metastasis in Osteosarcoma via Activation of the PI3K-Akt Signaling Pathway
}

\author{
Yong Chen ${ }^{a, b}$ Wending Huang ${ }^{a, b}$ Wei Sun Biqiang Zheng ${ }^{a, b}$ \\ Chunmeng Wang ${ }^{a, b}$ Zhiguo Luo ${ }^{b, c}$ Jian Wang ${ }^{b, d}$ Wangjun Yan ${ }^{a, b}$ \\ aDepartment of Musculoskeletal Oncology, Fudan University Shanghai Cancer, Shanghai, ${ }^{b}$ Department \\ of Oncology, Fudan University Shanghai Medical College, Shanghai, 'Department of Medical Oncology, \\ Fudan University Shanghai Cancer Center, Shanghai, dDepartment of Pathology, Fudan University \\ Shanghai Cancer Center, Shanghai, China
}

\section{Key Words}

Long non-coding RNA (IncRNA) - Metastasis-associated lung adenocarcinoma transcript 1 (MALAT1) • Osteosarcoma • Stemness $\bullet$ Proliferation • Metastasis • Prognosis

\begin{abstract}
Background/Aims: LncRNAs have been reported to be vital regulators of the progression of osteosarcoma, although the underlying mechanisms are not completely understood. Methods: The levels of MALAT1 and miR-129-5p expression were measured using qRT-PCR. Cell growth was determined using the CCK-8 and colony formation assays. Cell migration and invasion were detected using the wound healing and Transwell invasion assays, respectively. Tumor growth was determined with a xenograft model. Results: MALAT1 was significantly up-regulated in osteosarcoma tissues compared with adjacent non-tumor soft tissues. Overexpression of MALAT1 promoted osteosarcoma cell proliferation, migration, and invasion in vitro and enhanced tumor growth in a tumor xenograft mouse model. MALAT1 promoted osteosarcoma progression by modulating stem cell-like properties. Moreover, rescue experiment and luciferase reporter assay results indicated that MALAT1 modulates RET expression by sponging miR-129-5p in osteosarcomas. Furthermore, MALAT1 augmented the expression of downstream proteins of the RET-Akt pathway. MALAT1 was consistently significantly increased in osteosarcoma tissues and MALAT1 expression was positively correlated with tumor size and metastasis. High expression of MALAT1 was significantly associated with poor outcomes in patients with osteosarcomas. MALAT1 expression was positively related to RET and negatively related to miR-129-5p in osteosarcoma samples and xenograft tumors. MALAT1 functioned as an oncogenic IncRNA in osteosarcomas and was as an independent prognostic indicator. Conclusion: Our data revealed for the first time that
\end{abstract}

Y. Chen and W. Huang contributed equally to this work.

Yong Chen

and Wangjun Yan
Department of Musculoskeletal Oncology, Fudan University Shanghai Cancer,

Shanghai, 200032 (China)

E-Mail yongchenphd@163.com; dryanwangjun@sina.com 


\section{Cellular Physiology Cell Physiol Biochem 2018;51:1313-1326

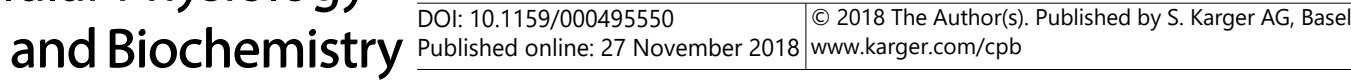 \\ Chen et al.: Role of LncRNA MALAT1 in Osteosarcoma}

MALAT1 increases stem cell-like properties by up-regulating RET via sponging miR-129-5p, and thus activates the PI3K-Akt signaling pathway and provides potential therapeutic targets for osteosarcoma treatment.

(C) 2018 The Author(s)

Published by S. Karger AG, Basel

\section{Introduction}

Osteosarcoma is the most common primary malignant bone tumor in children and adolescents worldwide [1-3]. In most cases, the tumors have metastasized to the lungs in patients diagnosed with osteosarcomas [4]. Standard neoadjuvant chemotherapy can effectively increase the possibility of limb salvage, which can significantly improve the 5 -year survival rate and quality of life in patients with osteosarcomas [5]; however, intrinsic or acquired chemotherapy resistance affects the therapeutic effect [6-8]. Previous studies have shown that chemotherapy resistance of tumors is a complex and multistage process, including genes, proteins, miRNAs, and signaling pathways $[9,10]$. Little is known about the molecular mechanism underlying chemotherapy resistance of osteosarcoma patients. Recent studies have focused on the role of lncRNAs in human tumors [11], which are noncoding RNAs with a length $>200$ nucleotides that differ from small non-coding RNAs, such as miRNA, tRNA, and rRNA [12]. Antisense lncRNAs are the reverse complementary sequences of endogenous RNA and account for approximately 60\% lncRNAs, which represents a large proportion of long chain non-coding transcriptomes [13]. MALAT1 can enhance the characteristics of glioma stem cells, which is related to the progression and poor prognosis of glioblastomas [14].

It has been shown that IncRNA MALAT1 is highly expressed in osteosarcomas and positively correlated with a poor prognosis [15]. In the current study we found that overexpression of MALAT1 in osteosarcomas was positively associated with prognosis and closely correlated with tumor stem cell characteristics, tumor invasion, and metastasis. MALAT1 blocked miR-129-5p by targeting the arginine kinase receptor of RET mRNA via a new mechanism. Activation of MALAT1 may thus serve as a potential prognostic biomarker and a therapeutic target for osteosarcoma.

\section{Materials and Methods}

\section{Samples}

Sixty-eight paired fresh primary osteosarcomas and corresponding adjacent non-tumor soft tissues were collected from tumor surgical resections performed at the Fudan University Shanghai Cancer from 2012-2015. All samples were immediately placed in liquid nitrogen and stored at $-80^{\circ} \mathrm{C}$. This study was approved by the Clinical Research Ethics Committee of Fudan University Shanghai Cancer. All of the human tissues were obtained with informed consent from the patients or their family members. Patients were followed every 3 months during the 1st year post-operatively until June 2017. Disease-free survival (DFS) was the time interval between tumor resection and recurrence, metastasis, death, or the last observation. The overall survival (OS) was the interval between the operation and death or the final follow-up evaluation.

\section{Cell lines}

Human osteosarcoma cell lines (MG63, U20S, Saos-2, SOSP-9607, and SW1353), normal cells (hFOB1.19), and fibroblast lines (NIH3T3 and 293T) were purchased from the American Type Culture Collection (ATCC; Manassas, VA, USA). All of the cell lines were cultured in DMEM medium containing 10\% fetal bovine serum (FBS, Thermo Fisher Scientific) and $100 \mathrm{U} / \mathrm{ml}$ of penicillin/streptomycin (Beyotime Biotechnology, China) at $37^{\circ} \mathrm{C}$ in a $5 \% \mathrm{CO}_{2}$ humidified incubator. 


\section{Cellular Physiology Cell Physiol Biochem 2018;51:1313-1326 \begin{tabular}{ll|l} 
and Biochemistry & $\begin{array}{l}\text { DOI: } 10.1159 / 000495550 \\
\text { Published online: } 27 \text { November } 2018\end{array}$ & $\begin{array}{l}\text { (c) } 2018 \text { The Author(s). Published by S. Karger AG, Basel } \\
\text { www.karger.com/cpb }\end{array}$
\end{tabular}

\section{Construction of lentivirus vector and a stable cell line}

Lentiviral vector encoding the full-length MALAT1 sequence was constructed by GeneCopoeia (Sangon, Shanghai, China). MALAT1 (shMALAT1) cDNA oligonucleotides were synthesized by Shanghai SBO Medical Biotechnology Company (Shanghai, China). Osteosarcoma cell lines (SW1353 and SOSP-9607) were transfected using Lipofectamine 2000 reagent (Invitrogen, Carlsbad, CA, USA) with lentivirus vector encoding the full-length MALAT1 sequence as the MALAT1 group; transfection with lentivirus vector and a shRNA blank served as the negative control (NC).

\section{Transient transfection}

One hundred nanomolar double-stranded miR-129-5p mimics, inhibitors, or negative control RNA (GenePharma, Shanghai, China) were transfected into the cells. The cells were collected $48 \mathrm{~h}$ after transfection. The procedure used was previously described [16].

Colony formation assay in vitro

After transfection, SW1353 and SOSP-9607 cells (1000 cells/ well) were inoculated into 6-well plates. After culturing for 7 days, 4\% paraformaldehyde was added for fixation and the plates were washed, dried, and the total clone number ( $>100$ cells/clone) was counted. The procedure used was previously described [17].

Wound healing assay and invasion assay in vitro

The migration ability of SW1353 and SOSP-9607 osteosarcoma cell lines was detected using a wound healing assay. The invasive ability was measured using the Transwell migration assay with a Transwell chamber (Corning, New York, USA) coated with Matrigel (Biosciences, Lincoln, NE). Two hundred microliters of a transfected SOSP-9607 (1×105 cells) or SW1353 $(2 \times 105)$ cell suspension in each group was used for the experiments. The procedure was previously described [18].

\section{Microsphere formation assay}

Transfected SW1353 or SOSP-9607 cells were inoculated into ultralow adherent 6-well plates (Corning). Ten thousand cells/well were cultured in $2 \mathrm{ml}$ of serum-free DMEM medium containing $20 \mathrm{mg} / \mathrm{L}$ of EGF (Corning Incorporated, Corning, NY, USA), $20 \mathrm{mg} / \mathrm{L}$ of hFGF (Peprotech), $4 \mathrm{U} / \mathrm{L}$ of insulin (SigmaAldrich, USA), and $100 \mathrm{U} / \mathrm{ml}$ of penicillin/streptomycin. The medium was changed every other day and the number of spheres was counted under a microscope (Leica, Germany).

\section{RNA isolation and $q R T-P C R$}

Total RNA was isolated using the TRIzol Reagent (Invitrogen) according to the manufacturer's protocol. Real-time PCR was performed on an ABI PRISM 7500 Fast Sequence Detection System (Li-Cor Biosciences, Lincoln, NE) using SYBR Green Supermix (GenePharma, Shanghai, China). PCR primers were as follows: MALAT1 F, 5'-ACGCAGGTGTGGCTTTCCAT-3' and R, 5'-GTCCTCCCTCACCACAATGG-3'; U6 F, 5'-GCGCTCTCTACTGGCATCACATA-3' and R, 5 -AATTTACGCTACGCTGTCTGCG-3'; and GAPDH F, 5'-GAATGCCTAGGTGAAACAGG-3' and R, 5'-TTAGGGTGAGCCAGATAGTG-3'. Gene expression was calculated using the $2^{-\Delta \Delta C \mathrm{t}}$ or $2^{-\triangle \mathrm{Ct}}$ method.

\section{Western blot}

Total protein was extracted from the transfected SW1353 and SOSP-9607 cells. After determination of the protein concentration, the protein was separated by SDS-PAGE electrophoresis and transferred to nitrocellulose membranes (Millipore, USA). AKT, RET, mTOR, 4EBP1, and P70S6K antibodies (Santa Cruz Biotech, Santa Cruz, CA) were added as primary antibodies and GAPDH was used as an internal reference control. After incubation with secondary antibodies, a chemiluminescent imaging system (ChemiDoc Touch; Bio-Rad, Hercules, CA, USA) was used to detect the signals. 


\section{Cellular Physiology Cell Physiol Biochem 2018;51:1313-1326

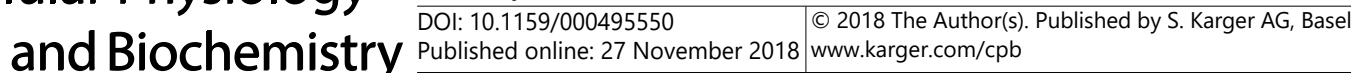 \\ Chen et al.: Role of LnCRNA MALAT1 in Osteosarcoma}

Flow cytometry assay (FCA) and fluorescence-activated cell sorting (FACS)

CD133-APC and CD44-PE antibodies were purchased from eBioscience (Bio-Rad). CD133 and CD44 antibodies (1:1000) were added to SW1353 and SOSP-9607 cells, respectively. The cells were incubated in PBS containing $1 \%$ fetal bovine serum at $4^{\circ} \mathrm{C}$. After washing with PBS, the cells were detected by Cyto FLEX (Beckman, Germany) and the results were analyzed by Cyt Expert (Amersham Biosciences, Buckinghamshire, UK). When fluorescence-activated cells were sorted, cells labeled with CD133-APC and CD44-PE (BD Influx; Biosciences, Lincoln, NE) were separated. The unstained cells were used as NCs.

Nude mouse model in vivo

Male BALB/C nude mice (6-8 weeks old; weight,18-20 g) were fed at the Experimental Animal Center of Fudan University Shanghai Cancer with SPF facilities. Mice were randomly divided into 3 groups (MALAT1, sh-MALAT1, and NC). One hundred microliters of SW1353 cells $\left(2 \times 10^{6}\right)$ were injected subcutaneously. On the 21st day after implantation, the mice in the subcutaneous group received euthanasia and the tumors were resected. Tumor tissues were weighed and partially processed for qRT-PCR.

Fluorescence in situ hybridization (FISH) of RNA

According to the instructions, FISH was performed on 24-well plates using a FISH Kit (Ribobio, China). The staining method was performed as previously described [19].

\section{Cell fractionation assay}

The cytoplasm and nucleus RNA were obtained using a RNA Purification Kit (Norgen, Thorold, ON, Canada). Cells and tumor tissues were collected, then lysate was added. After incubation for 5 min on ice, the cells were centrifuged at $12000 \mathrm{~g}$ for $3 \mathrm{~min}$. The supernatant was collected to obtain cytoplasmic RNA and nuclear RNA was extracted using nucleus beads.

\section{Luciferase reporter assay}

The following 4 vectors of pmiR-RB-REPORT ${ }^{\mathrm{TM}}$ were chemically synthesized: RET 3'-UTR containing the putative target site for miR-129-5p (RET WT-3'-UTR); RET 3'-UTR containing the mutation binding site (RET Mut-3'-UTR); full-length MALAT1 containing the putative target site for miR-129-5p (MALAT1-WT); and full-length MALAT1 containing the mutation binding site (MALAT1-Mut). One hundred nanograms of vector (RET WT-3'-UTR, RET Mut-3'-UTR, MALAT1-WT, or MALAT1-Mut) and miR-129-5p mimics or mimics control ( $50 \mathrm{nM}$ /well) were co-transfected into $293 \mathrm{~T}$ and SW1353 cells using riboFECT ${ }^{\mathrm{TM}}$ CPRibobio reagent (Ribobio). Mimics control and RET Mut-3'UTR were used as NCs. The luciferase activity was detected as previously described [20].

\section{Statistical analysis}

Statistical analysis was performed using SPSS 15 software (SPSS, Inc., Chicago, IL, USA). Data were compared with a two-tailed Student's t-test, Wilcoxon rank-sum test, Fisher's exact test, one-way ANOVA, or Kruskal-Wallis test. DFS and OS were calculated and a multivariate Cox proportional hazards model was used to analyze the independent factors. Survival curves were drawn using the Kaplan-Meier method, and a logarithmic rank test was used to compare survival rates. Spearman and Pearson correlation analyses were used to detect correlations. A $\mathrm{P}<0.05$ was considered a significant difference.

\section{Results}

MALAT1 promotes proliferation, migration, and invasion in osteosarcoma cells in vitro Compared with hFOB1.19 or NIH3T3 cells, the expression of MALAT1 in osteosarcoma cells increased significantly (Fig. 1A). The results from qRT-PCR showed that compared with the NC group, the expression of MALAT1 in SW1353 and SOSP-9607 cells was up-regulated in the MALAT1 group, but significantly down-regulated in the shMALAT1 group (Fig. 1B). Overexpression of MALAT1 significantly increased the clone numbers in SW1353 and SOSP9607 cells (Fig. 1C). Overexpression of MALAT1 significantly increased the migration ability of SW1353 and SOSP-9607 cells, whereas knockdown of MALAT1 significantly reduced the migration ability of these cells (Fig. 1D, E). The Transwell invasion assay showed 


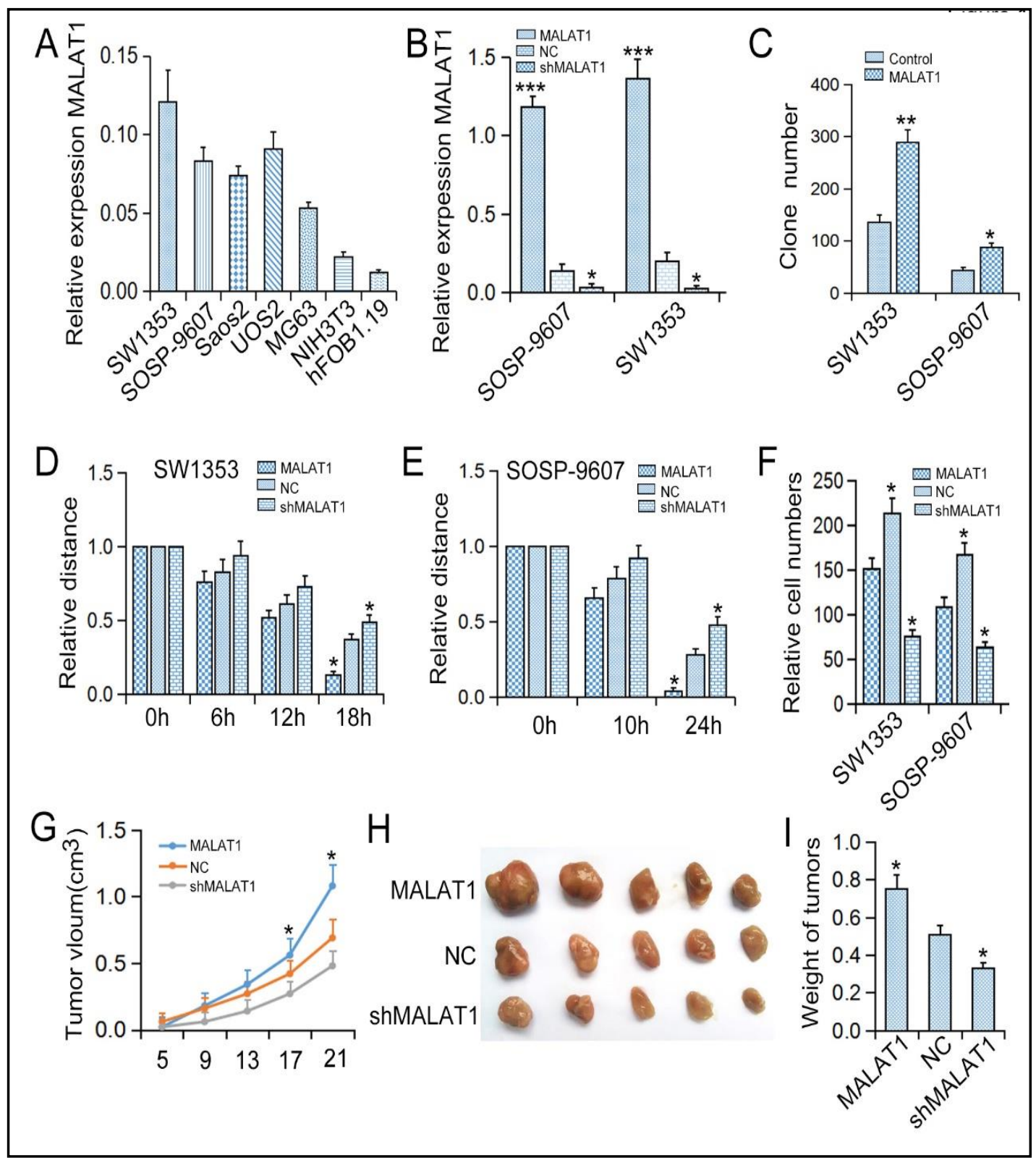

Fig.1. MALAT1 promotes proliferation, migration, and invasion of osteosarcoma cells. (A) Compared with hFOB1.19 and NIH3T3 cells, the expression of MALAT1 increased significantly in osteosarcoma cell lines $\left({ }^{*} \mathrm{P}<0.05\right)$; (B) The transfection efficiency was detected by qRT-PCR. Compared with the blank vector group, the expression of MALAT1 was significantly up-regulated in MALAT1-transfected SW1353 and SOSP9607 cells, while MALAT1 in shRNA group was significantly down-regulated $\left({ }^{*} \mathrm{P}<0.05, * * * \mathrm{P}<0.001\right)$; (C) Colony formation assay in SW1353 and SOSP-9607 cells showed that the number of clones in the MALAT1 overexpressing cells increased significantly compared with the control cells, while the number of clones in the MALAT1 knockdown cells decreased significantly; (D) Cell migration assay detected the migration ability of SW1353 cells. Overexpression of MALAT1 decreased cell migration distance significantly $\left({ }^{*} \mathrm{P}<0.05\right) ;(\mathrm{E})$ The migration ability of SOSP-9607 cells detected by cell migration assay $\left({ }^{*} \mathrm{P}<0.05\right)$; $(\mathrm{F})$ Transwell assay showed that the invasive potential of SW1353 and SOSP-9607 was significantly increased upon MALAT1 overexpression $\left({ }^{*} \mathrm{P}<0.05\right)$; $(\mathrm{G}-\mathrm{I})$ Volume, size, and weight of xenografts in each group $\left({ }^{*} \mathrm{P}<0.05\right)$. 
that the invasive ability of osteosarcoma cells was increased significantly after MALAT1 overexpression, but remarkably decreased upon MALAT1 knockdown (Fig. 1F). These results suggested that MALAT1 promotes the proliferation, migration, and invasion of osteosarcoma cells in vitro.

\section{MALAT1 promotes tumorigenicity of osteosarcomas in vivo}

Although all mice in the MALAT1, sh-MALAT1, and NC groups bore tumors, 3 weeks after injection, the tumor volume in the MALAT1 group increased significantly compared with the NC group (Fig. 1G, H). In contrast, the tumor volume in the shMALAT1 group was significantly decreased (Fig. 1G, H). In addition, compared with the NC group the tumor weight in the MALAT1 group increased significantly, while the opposite change was observed in the shMALAT1 group (Fig. 1I).

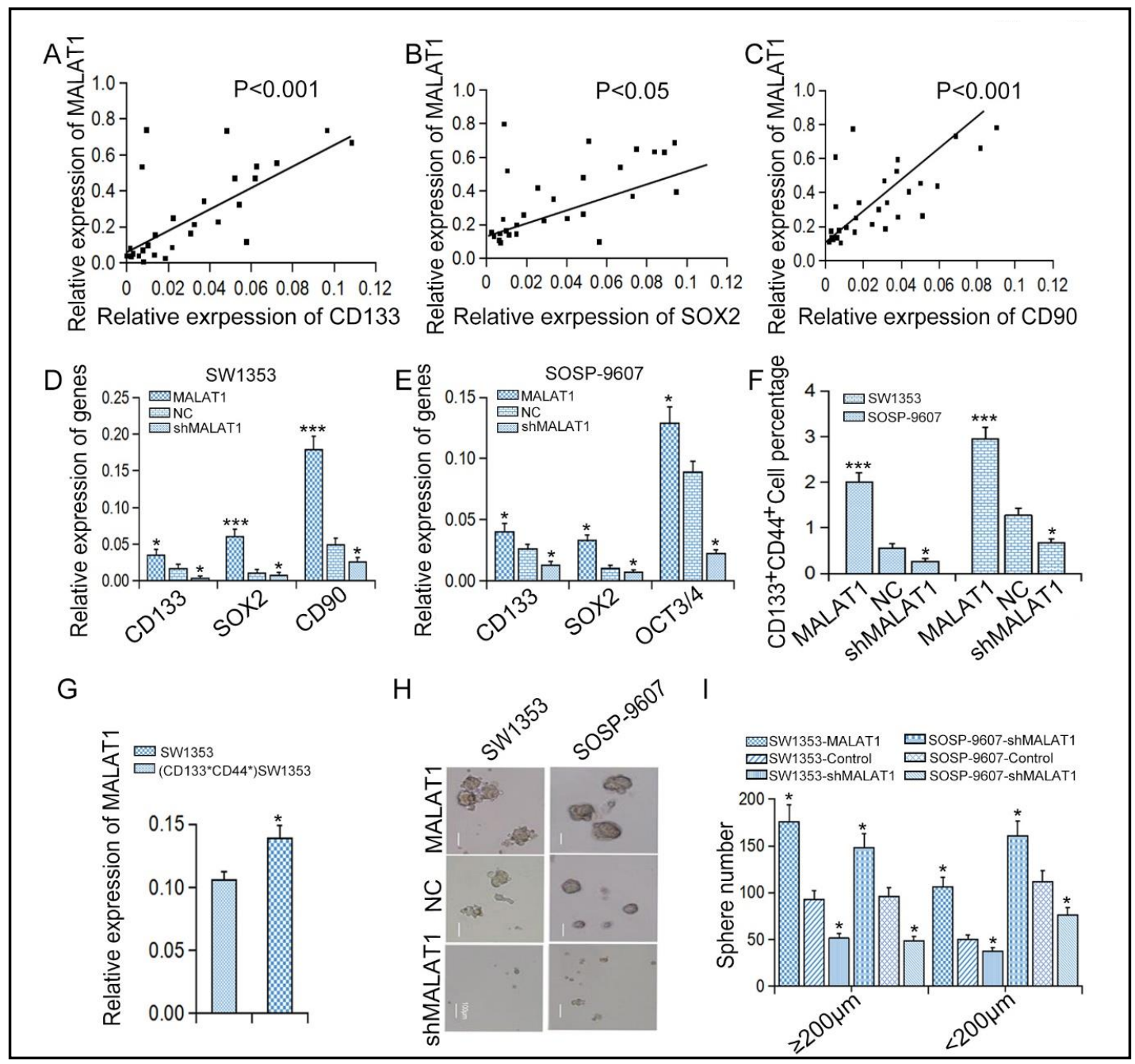

Fig. 2. MALAT1 promotes the characteristics of osteosarcoma stem cells. In osteosarcoma tissues, MALAT1 and stem cell markers were co-expressed (A-C) and compared with ncMALAT1 cells. MALAT1 overexpression up-regulated the expression of CD133, CD90, and SOX2 in SW1353 (D) and SOSP-9607 cells (E), while sh-MALAT1 down-regulated the expression of the above factors; (F) The results of FCA showed that overexpression of MALAT1 also increased the proportion of CD133+/CD44+ cells in SW1353 and SOSP9607 cells, while down-regulation of MALAT1 decreased the proportion of CD133+/CD44+ cells $\left({ }^{*} \mathrm{P}<0.05\right.$, *** $\mathrm{P}<0.001$ ); (G) Compared with SOSP-9607 cell populations, the expression of MALAT1 in CD133+/CD44+ cells increased significantly $\left({ }^{*} \mathrm{P}<0.05\right)$; $(\mathrm{H})$ Photographs of tumor microspheres in different groups (scale, $100 \mathrm{~mm}$ ); (I) The statistical histogram of microsphere formation showed that overexpression of MALAT1 increased the tumor stem cell microsphere number of SW1353 and SOSP-9607 cells. 


\section{$\begin{array}{lll}\text { Cellular Physiology } & \text { Cell Physiol Biochem 2018;51:1313-1326 } \\ \text { Dol: 10.1159/000495550 } & \text { (O2018 The Author(s). Published by S. Karger AG, Basel }\end{array}$

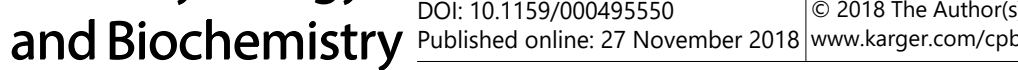

Correlation between MALAT1 expression and tumor stem cell-like properties

The qRT-PCR results showed that MALAT1 was significantly correlated with the expression of biomarkers of stemness (CD133, CD90, and SOX2) in osteosarcoma tissues (Fig. 2A-C). In agreement with these results, the expression of CD133, CD90, and SOX2 mRNA was significantly up-regulated in MALAT1 overexpression group compared with the NC group (Fig. 2D, E), but significantly down-regulated in the shMALAT1 group (Fig. 2D, E). FCA analysis showed that ectopic expression of MALAT1 increased the CD133 ${ }^{+} \mathrm{CD} 44^{+}$ cell ratio, while down-regulation of MALAT1 reduced the ratio of these stem cells (Fig. 2F). Moreover, the expression of MALAT1 was enriched in $\mathrm{CD} 133^{+} \mathrm{CD} 44^{+}$cells compared with the NC group (Fig. 2G). In addition, the microsphere formation assay showed that MALAT1 overexpression significantly increased tumor microsphere formation, whereas MALAT1 knockdown significantly inhibited the number and size of tumor microspheres in both SW1353 and SOSP-9607 cells (Fig. 2H, I). These results indicated that MALAT1 enhanced the population of osteosarcoma stem cells.

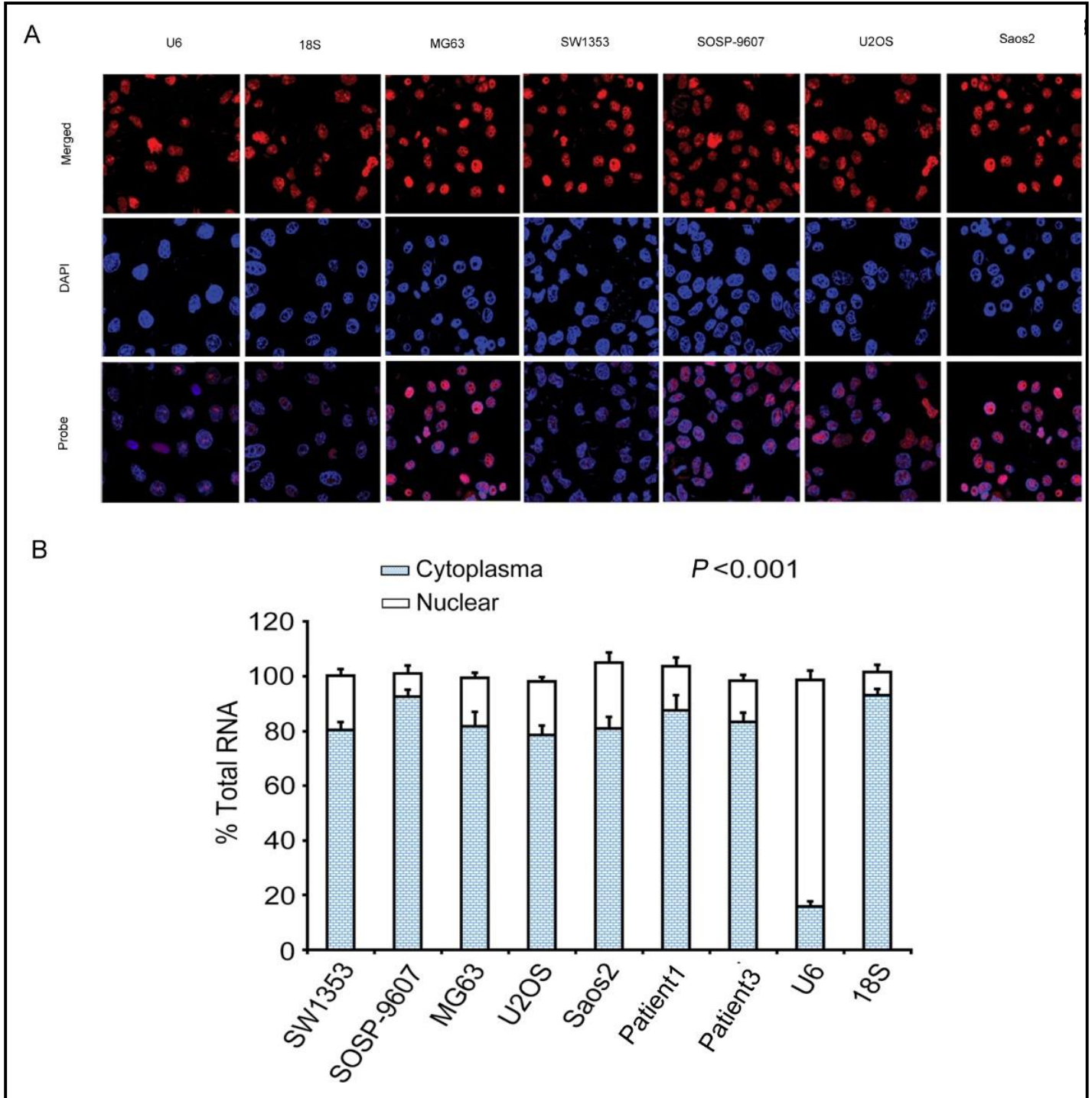

Fig.3. MALAT1 is a post-transcriptional regulatory factor. (A) The results of RNA FISH showed that MALAT1 was mainly located in the cytoplasm of SW1353, SOSP-9607, MG63, U20S, and Saos2 osteosarcoma cell lines; (B) The cell component results by qRT-PCR showed that MALAT1 was mainly located in the cytoplasm of osteosarcoma cells and in the tumor tissues of two patients. 
Fig. 4. MALAT1 upregulates RET through competitive binding with miR-129-5p and activation of the AKT signaling pathway. (A) Predicted binding site for miR-129-5p in MALAT1; (B) Predicted binding site for miR-129-5p in RET 3'-UTR; (C) Luciferase activity in 293T cells after MALAT1-WT or MALAT1Mut vector co-transfected with miR-129-5p mimics. MiR-129-5p mimics reduced the luciferase activity of MALAT1-WT vector, but did not reduce the luciferase activity of the blank vector or the mutant reporter; (D) The luciferase activity of MALAT1 and miR-129$5 p$ in SW1353 cells was similar to that of 293T cells; (E) Luciferase activity in 293T cells after RET-WT or RET-Mut vector co-transfected with miR-129-5p mimics. MiR129-5p mimics reduced the luciferase activity of RET-WT vector, but did not reduce the luciferase activity of RET mutation reporter; (F) The luciferase activity of RET and miR129-5p in SW1353 cells was similar to that of 293T cells; (G) qRT-PCR results showed that RET expression was higher in MALAT1-overexpressing SW1353 and SOSP9607 cells compared with control cells; (H) The results of qRT-PCR showed the expression of miR-129-5p in ectopic

MALAT1-transfected cells decreased significantly compared with control cells; (I) The results of qRT-PCR showed miR-129-5p inhibitor significantly increased RET expression in SW1353 and SOSP-9607 cells, while miR-129-5p mimics reduced RET expression; (J) The results of Western blot showed the expression pattern of the MALAT1-RET-AKT signaling pathway in osteosarcoma cells.

B

MALAT1-WT 5' -UUUGGGUGGaAUGCAAAAAU-3' miR-129-5p 3'-CGUUCGGGUCUGGCGUUUUUUC-5' MALAT1-MUT $\quad 5^{\prime}$-UUUGGGUGGGAAUCGUUUUUUU-3'
C

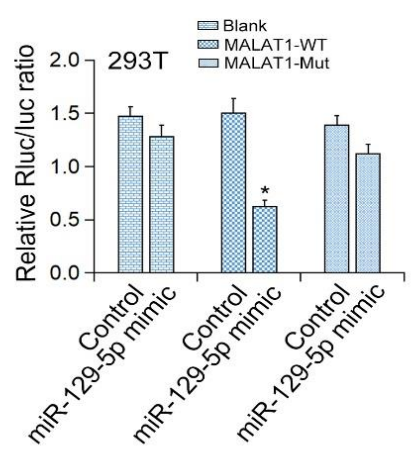

E

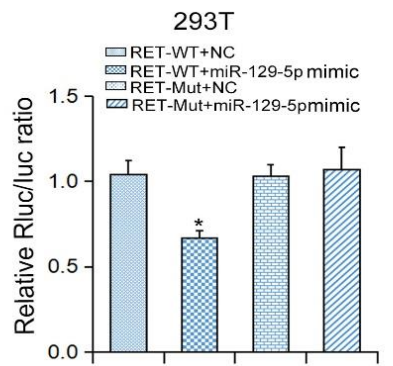

G

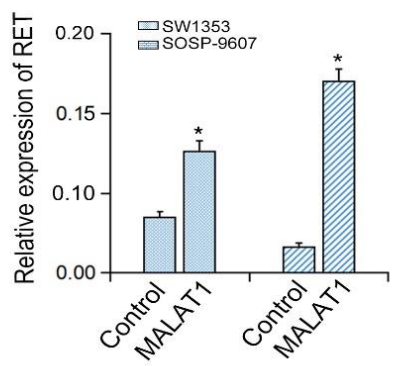

I

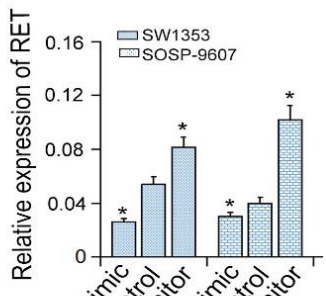

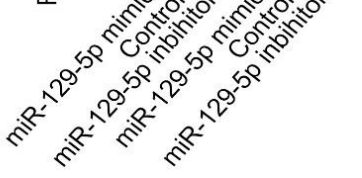

RET WT-3'-UTR 5' -GCCGCCAAACAAGGCAAAAAA-3' miR-129-5p 3'-CGUUCGGGUCUGGCGUUUUUUC-5 RET MUt-3'-UTR 5' -GCCGCCAAACAAGCGUUUUUUA-3'

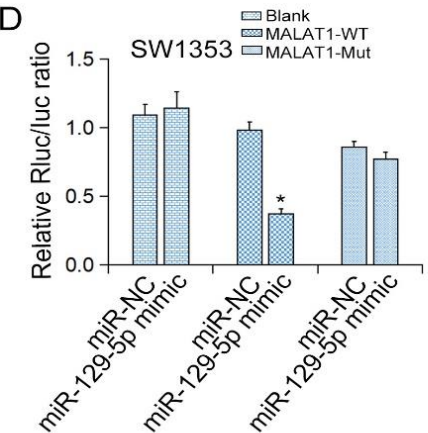

$\mathrm{F}$

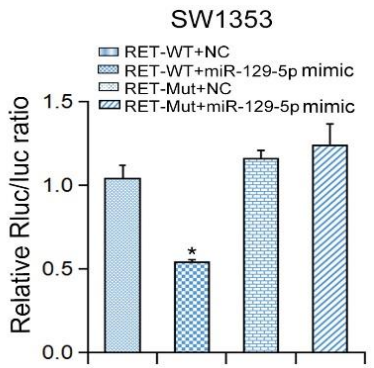

$\mathrm{H}$

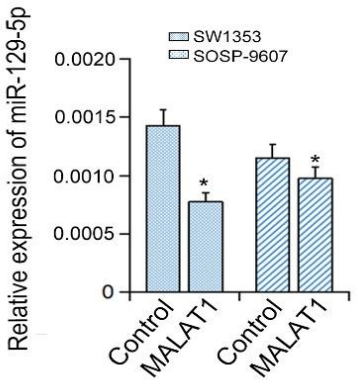

J

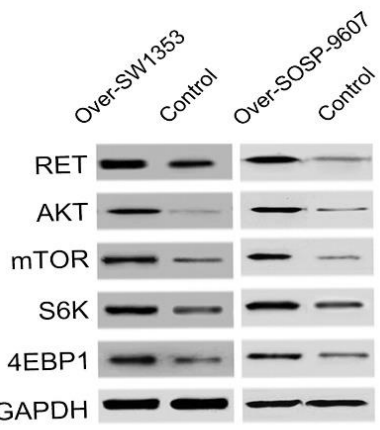




\section{Cellular Physiology Cell Physiol Biochem 2018;51:1313-1326

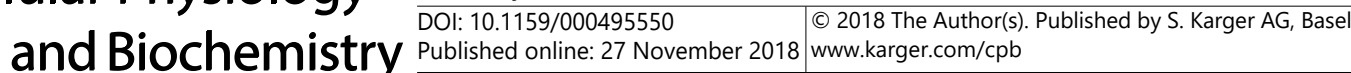 \\ Chen et al.: Role of LncRNA MALAT1 in Osteosarcoma}

\section{MALAT1 negatively targets miR-129-5p}

The FISH results showed that MALAT1 is mainly located in the cytoplasm of osteosarcoma cells (Fig. 3A). The cellular fractionation assay also showed that MALAT1 is highly expressed in the cytoplasm (Fig. 3B). To determine whether or not MALAT1 competitively combines with microRNA to function as a post-transcriptional regulator, we used the Starbase database (http://starbase.sysu.edu.cn/index.php) to analyze the interaction. The predicted result showed that MALAT1 combined with miR-129-5p with a high alignment score. In addition, according to the prediction of the TargetScan database (http://www.targetscan. org/vert_72/), miR-129-5p bound to the 3'-UTR of RET mRNA.

MALAT1 up-regulated RET by competitively binding with miR-129-5p and triggerred the Akt signaling pathway

To validate the direct binding site between MALAT1 and miR-129-5p, we performed luciferase reporter assays. MiR-129-5p mimics reduced the luciferase activity of the MALAT1WT reporter vector, but had no effect on the luciferase activity of the blank vector in 293T and SW1353 cells (Fig. 4A, C, D). To determine the binding site between miR-129-5p and RET 3'-UTR, we constructed a luciferase RET WT-3'-UTR reporter vector containing the miR-129$5 p$ binding site and a RET Mut-3'-UTR reporter vector containing the miR-129-5p mutation binding site (Fig. 4B). Similarly, miR-129-5p mimics reduced the luciferase activity of the RET WT-3'-UTR reporter vector, but did not reduce the luciferase activity of RET Mut-3'-UTR reporter vector in 293T and SW1353 cells (Fig. 4E, F). Moreover, compared with the control group, RET mRNA was highly expressed in MALAT1-overexpressing SW1353 and SOSP-9607 cells (Fig. 4G). In contrast, the expression of miR-129-5p in ectopic MALAT1-transfected cells were significantly decreased compared with the NC group (Fig. 4H). In addition, miR-129-5p inhibitors significantly increased RET mRNA expression in SW1353 and SOSP-9607 cells, while miR-129-5p mimics reduced RET mRNA expression (Fig. 4I). Therefore, we assumed that MALAT1 competitively bound to miR-129-5p to block the inhibition of RET expression mediated by miR-129-5p. The RET protein level in the ectopic MALAT1-transfected cells was higher compared with the control cells, and the protein expression in the downstream of PI3K-Akt pathway was also increased after MALAT1 overexpression (Fig. 4J). These results indicated that MALAT1 up-regulated RET expression by competitively binding with miR129-5p, and hence triggered the Akt signaling pathway.

\section{MALAT1 promoted cell proliferation and migration by modulating the miR-129-5p/RET} pathway

To verify whether or not MALAT1 exerts an oncogenic effect by modulating the miR-1295p/RET pathway, we transfected siRNA RET (si-RET) or miR-129-5p mimics into the control cells or MALAT1-overexpressing SW1353 and SOSP-9607 cells. Compared with the control cells, the clone number of SW1353 and SOSP-9607 cells transfected with si-RET or miR-129$5 \mathrm{p}$ mimics decreased significantly, while overexpression of MALAT1 partially counteracted this decline (Fig. 5A, B). Similarly, si-RET or miR-129-5p mimics also reduced the migration (Fig. 5C) and invasion (Fig. 5D) of SW1353 and SOSP-9607 cells, while overexpression of MALAT1 diminished these changes. Furthermore, qRT-PCR showed that the mRNA level of RET in the MALAT1-overexpressing xenografts was significantly higher than the control xenograft, while RET mRNA expression in the shMALAT1 xenograft was decreased (Fig. 5E). In contrast, the expression of miR-129-5p in different xenograft groups was reversed (Fig. $5 \mathrm{~F})$. These results suggested that MALAT1 promotes osteosarcoma cell proliferation and migration through the miR-129-5p/RET pathway.

\section{MALAT1 predicted poor prognosis in osteosarcoma}

To explore the clinical significance of MALAT1, we determined the expression of MALAT1 in osteosarcoma samples. qRT-PCR results showed that MALAT1 expression in osteosarcoma tissues was significantly higher compared with the adjacent para-carcinoma tissues (Fig. 6A). MALAT1 expression was higher in the metastasis group compared to the non-metastasis

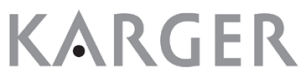






Fig. 5. The relationship between MALAT1, RET, and miR-129-5p in osteosarcoma cell lines and xenografts. (A-B) The results of colony formation showed that the number of clones decreased significantly in the RETsiRNA or miR-129-5p mimics-transfected cells, while MALAT1 overexpression partly reduced the colony number of SW1353 and SOSP-9607 cells ( $\left.{ }^{*} \mathrm{P}<0.05\right)$; 1:Control, 2:RET siRNA, 3:MALAT1+RET siRNA, 4: miR-129-5p mimic, 5: MALAT1+miR-129-5p mimic; (C-D) The results of cell migration showed that the cell migration distance increased in the RET-siRNA or miR-129-5p mimics-transfected SW1353 and SOSP9607 cells, indicating that the migration ability was inhibited. Overexpression of MALAT1 counteracted this inhibition $\left({ }^{*} \mathrm{P}<0.05\right)$; (E) Compared with the control xenografts, the expression of RET mRNA increased significantly in the MALAT1-overexpressing xenografts, while RET mRNA expression decreased in the shMALAT1 xenografts $\left({ }^{*} \mathrm{P}<0.05\right)$; (F) The expression of miR-129-5p in the MALAT1-overexpressing group decreased significantly compared with the control group $\left({ }^{*} \mathrm{P}<0.05\right)$, while miR-129-5p expression increased significantly in the shMALAT1 xenografts $(* * * \mathrm{P}<0.01)$. 


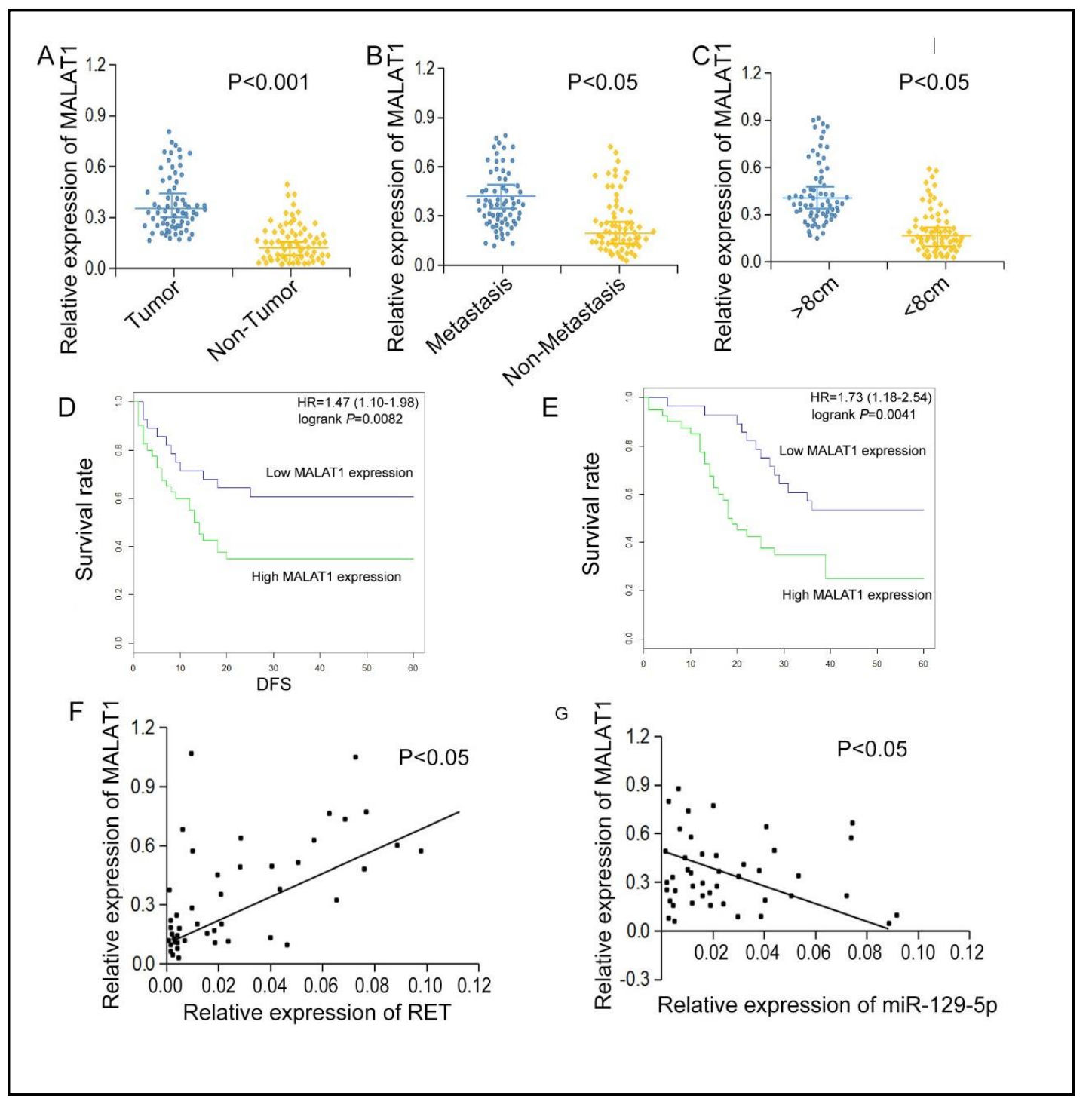

Fig. 6. The expression of MALAT1 and the relationship between MALAT1, RET, and miR-129-5 in osteosarcoma tissues. (A) The results of qRT-PCR showed that the expression of MALAT1 in osteosarcoma tissues increased significantly compared with adjacent normal tissues in 68 patients $\left({ }^{* * *} \mathrm{P}<0.001\right)$; (B) The expression of MALAT1 in tumor tissues with metastasis was significantly higher than tumor tissues without metastasis $\left({ }^{*} \mathrm{P}<0.05\right)$; (C) Compared with tumors $<8 \mathrm{~cm}$, the expression of MALAT1 increased significantly in tumors $>8 \mathrm{~cm}\left({ }^{*} \mathrm{P}<0.05\right)$; $(\mathrm{D}, \mathrm{E})$ DFS and OS were shorter in patients with higher MALAT1 expression by Kaplan-Meier analysis ( $\left.{ }^{* *} \mathrm{P}<0.001\right)$; (F) The correlation between RET mRNA and MALAT1 $\left({ }^{*} \mathrm{P}<0.05\right)$; $(\mathrm{G})$ miR-129-5p was negatively correlated with MALAT1 expression in osteosarcoma tissues $\left({ }^{*} \mathrm{P}<0.05\right)$.

group (Fig. 6B). MALAT1 expression was higher in specimens with a tumor volume $>8 \mathrm{~cm}$ compared with a tumor volume $<8 \mathrm{~cm}$ (Fig. 6C). Kaplan-Meier analysis indicated that DFS and OS were shorter in patients with higher MALAT1 expression (Fig. 6D, E). Additionally, the expression of RET was positively correlated with MALAT1 in the osteosarcoma group (Fig. 6F), while miR-129-5p was negatively correlated with MALAT1 (Fig. 6G). Moreover, Cox proportional hazards regression analysis showed that the expression of MALAT1 and RET were independent prognostic indicators of osteosarcoma (Table 1). Taken together, these results suggested that MALAT1 may be the key carcinogenic regulator and can promote the occurrence and progression of osteosarcoma. 


\section{Cellular Physiology Cell Physiol Biochem 2018;51:1313-1326 \begin{tabular}{ll|l} 
and Biochemistry Published online: 27 November 2018 & $\begin{array}{l}\text { (c) } 2018 \text { The Author(s). Published by S. Karger AG, Basel } \\
\text { www.karger.com/cpb }\end{array}$ \\
\hline
\end{tabular} \\ Chen et al.: Role of LncRNA MALAT1 in Osteosarcoma}

Table 1. Multivariable Cox's regression analysis of prognostic factors for osteosarcoma patients

\begin{tabular}{lllll}
\hline Clinicopathological parameters & Disease-free survival hazard ratio $(95 \% \mathrm{CI})$ & $P$ & Overall survival hazard ratio $(95 \% \mathrm{CI})$ & $P$ \\
\hline MALAT1 expression (high vs low) & $0.254(0.058-0.856)$ & 0.027 & $0.169(0.039-0.628)$ & 0.006 \\
RET expression (high vs low) & $1.551(0.449-4.631)$ & 0.028 & $6.024(1.104-10.075)$ & 0.027 \\
miR-129-5p expression (high vs low) & $0.401(0.175-1.208)$ & 0.014 & $0.806(0.503-1.577)$ & 0.008 \\
Tumor size (>8 cm vs < $8 \mathrm{~cm})$ & $1.068(0.627-1.834)$ & 0.573 & $0.361(0.142-1.195)$ & 0.031 \\
Metastasis (Positive vs negative) & $0.025(0.004-0.205)$ & 0.001 & $0.152(0.048-0.512)$ & 0.001 \\
\hline
\end{tabular}

\section{Discussion}

More and more evidence has shown that lncRNA plays an important role in the occurrence and progression of diseases [21]. A series of IncRNAs have been annotated and reported to have important roles $[22,23]$. The current study revealed that lncRNA MALAT1 expression was dysregulated in osteosarcoma and was related to patient survival time and could be an independent predictor of prognosis. The results showed that MALAT1 overexpression promotes proliferation, migration, and invasion of osteosarcoma cells in vitro, while inhibition of MALAT1 had an opposing effect. Moreover, MALAT1 ectopic expression promoted tumor growth in xenografts.

Similar to the functions of other lncRNAs, we found that MALAT1 expression was significantly associated with stem cell characteristic gene expression in osteosarcoma tissues. Overexpression of MALAT1 significantly increased the expression of CD133, SOX2, and CD90 in osteosarcoma cells. After stable overexpression of MALAT1, the proportion of CD133+CD44+ cells increased significantly, and the expression of MALAT1 in CD133+CD44+ cells was also up-regulated. In addition, MALAT1 overexpression increased the number and size of stem-cell like osteosarcoma microspheres. In summary, MALAT1 expression promotes the occurrence and progression of osteosarcomas by promoting the formation of osteosarcoma stem cells. Our data suggest that MALAT1 is mainly located in the cytoplasm, indicating that MALAT1 is a post-transcriptional regulator of osteosarcoma cells.

Cuccuru et al. [24] concluded that the RET gene is an important oncogene. Thus, we reasoned that the RET gene and RET-encoded products may be potential therapy targets for osteosarcomas. Therefore, it is necessary to determine whether or not there is a regulatory network between MALAT1 and RET. Interestingly, although MALAT1 does not bind with RET mRNA directly, MALAT1 and RET mRNA contain the same putative miR-129-5p binding sequence. Moreover, there is a correlation between MALAT1, RET, and miR-129-5p in osteosarcoma cells and tumor tissues. We also showed that MALAT1 overexpression is positively related to RET mRNA and negatively related to miR-129-5p, suggesting that there is a regulatory network in osteosarcoma cells. Our results also indicated that up-regulation of MALAT1 is a key factor in osteosarcomas and an independent prognostic predictor.

\section{Conclusion}

Taken together, MALAT1 is up-regulated in osteosarcoma cells and tissues and mediates tumor stem cell characteristics. MALAT1 competitively combines with miR-129-5p, which results in up-regulation of RET and activation of the Akt pathway, leading to osteosarcoma cell proliferation and promotion of migration. Our findings construct a new regulatory network in osteosarcomas that can serve as a potential therapeutic target. 


\section{Cellular Physiology Cell Physiol Biochem 2018;51:1313-1326 \begin{tabular}{ll|l} 
and Biochemistry & $\begin{array}{l}\text { DOI: 10.1159/000495550 } \\
\text { Published online: } 27 \text { November } 2018\end{array}$ & $\begin{array}{l}\text { (c) } 2018 \text { The Author(s). Published by S. Karger AG, Basel } \\
\text { www.karger.com/cpb }\end{array}$ \\
\hline
\end{tabular} \\ Chen et al.: Role of LncRNA MALAT1 in Osteosarcoma}

\section{Abbreviations}

Long (non-coding RNA, lncRNA); metastasis-associated (lung adenocarcinoma transcript 1, MALAT1); overall (survival, OS); disease-free (survival, DFS); flow (cytometry assay, FCA); fluorescence-activated (cell sorting, FACS); fluorescence (in situ hybridization, FISH).

\section{Acknowledgements}

This work was supported by National Natural Science Foundation of China (NSFC) (Grant No. 81302342).

\section{Disclosure Statement}

The authors declare that they have no competing interests.

\section{References}

1 Isakoff MS, Bielack SS, Meltzer P, Gorlick R: Osteosarcoma: Current Treatment and a Collaborative Pathway to Success. J Clin Oncol 2015;33:3029-3035.

-2 Sottnik JL, Campbell B, Mehra R, Behbahani-Nejad O, Hall CL, Keller ET: Osteocytes serve as a progenitor cell of osteosarcoma. J Cell Biochem 2014;115:1420-1429.

-3 Di Fiore R, Guercio A, Puleio R, Di Marco P, Drago-Ferrante R, D’Anneo A, De Blasio A, Carlisi D, Di Bella S, Pentimalli F, Forte IM, Giordano A, Tesoriere G, Vento R: Modeling human osteosarcoma in mice through 3AB-OS cancer stem cell xenografts. J Cell Biochem 2012;113:3380-3392.

4 Shaikh AB, Li F, Li M, He B, He X, Chen G, Guo B, Li D, Jiang F, Dang L, Zheng S, Liang C, Liu J, Lu C, Liu B, Lu J, Wang L, Lu A, Zhang G: Present Advances and Future Perspectives of Molecular Targeted Therapy for Osteosarcoma. Int J Mol Sci 2016;17:506.

5 Del Mare S, Husanie H, Iancu 0, Abu-Odeh M, Evangelou K, Lovat F, Volinia S, Gordon J, Amir G, Stein J, Stein GS, Croce CM, Gorgoulis V, Lian JB, Aqeilan RI: WWOX and p53 Dysregulation Synergize to Drive the Development of Osteosarcoma. Cancer Res 2016;76:6107-6117.

6 Jiang B, Mu W, Wang J, Lu J, Jiang S, Li L, Xu H, Tian H: MicroRNA-138 functions as a tumor suppressor in osteosarcoma by targeting differentiated embryonic chondrocyte gene 2. J Exp Clin Cancer Res 2016;35:69.

7 Wang L, Yang L, Lu Y, Chen Y, Liu T, Peng Y, Zhou Y, Cao Y, Bi Z, Liu T, Liu Z, Shan H: Osthole Induces Cell Cycle Arrest and Inhibits Migration and Invasion via PTEN/Akt Pathways in Osteosarcoma. Cell Physiol Biochem 2016;38:2173-2182.

>8 Li Y, Wagner ER, Yan Z, Wang Z, Luther G, Jiang W, Ye J, Wei Q Wang J, Zhao L, Lu S, Wang X, Mohammed MK, Tang S, Liu H, Fan J, Zhang F, Zou Y, Song D, Liao J et al.: The Calcium-Binding Protein S100A6 Accelerates Human Osteosarcoma Growth by Promoting Cell Proliferation and Inhibiting Osteogenic Differentiation. Cell Physiol Biochem 2015;37:2375-2392.

-9 Kareva I, Waxman DJ, Lakka Klement G: Metronomic chemotherapy: an attractive alternative to maximum tolerated dose therapy that can activate anti-tumor immunity and minimize therapeutic resistance. Cancer Lett 2015;358:100-106.

10 Laurila N, Koivunen JP: EGFR inhibitor and chemotherapy combinations for acquired TKI resistance in EGFR-mutant NSCLC models. Med Oncol 2015;32:205.

11 Liu Y, Sharma S, Watabe K: Roles of lncRNA in breast cancer. Front Biosci (Schol Ed) 2015;7:94-108.

12 Sanchez Y, Segura V, Marin-Bejar O, Athie A, Marchese FP, Gonzalez J, Bujanda L, Guo S, Matheu A, Huarte M: Genome-wide analysis of the human p53 transcriptional network unveils a lncRNA tumour suppressor signature. Nat Commun 2014;5:5812. 


\section{Cellular Physiology Cell Physiol Biochem 2018;51:1313-1326

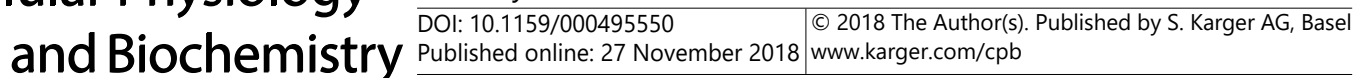 \\ Chen et al.: Role of LncRNA MALAT1 in Osteosarcoma}

13 Guo Q Cheng Y, Liang T, He Y, Ren C, Sun L, Zhang G: Comprehensive analysis of IncRNA-mRNA coexpression patterns identifies immune-associated lncRNA biomarkers in ovarian cancer malignant progression. Sci Rep 2015;5:17683.

14 Li Z, Xu C, Ding B, Gao M, Wei X, Ji N: Long non-coding RNA MALAT1 promotes proliferation and suppresses apoptosis of glioma cells through derepressing Rap1B by sponging miR-101. J Neurooncol 2017;134:19-28.

15 Wang Y, Zhang Y, Yang T, Zhao W, Wang N, Li P, Zeng X, Zhang W: Long non-coding RNA MALAT1 for promoting metastasis and proliferation by acting as a ceRNA of miR-144-3p in osteosarcoma cells. Oncotarget 2017;8:59417-59434.

16 Zhang EB, Yin DD, Sun M, Kong R, Liu XH, You LH, Han L, Xia R, Wang KM, Yang JS, De W, Shu YQ Wang ZX: P53-regulated long non-coding RNA TUG1 affects cell proliferation in human non-small cell lung cancer, partly through epigenetically regulating HOXB7 expression. Cell Death Dis 2014;5:e1243.

17 Huang MD, Chen WM, Qi FZ, Sun M, Xu TP, Ma P, Shu YQ: Long non-coding RNA TUG1 is up-regulated in hepatocellular carcinoma and promotes cell growth and apoptosis by epigenetically silencing of KLF2. Mol Cancer 2015;14:165.

18 Shi SJ, Wang LJ, Yu B, Li YH, Jin Y, Bai XZ: LncRNA-ATB promotes trastuzumab resistance and invasionmetastasis cascade in breast cancer. Oncotarget 2015;6:11652-11663.

19 Chen L, Yang H, Xiao Y, Tang X, Li Y, Han Q, Fu J, Yang Y, Zhu Y: Lentiviral-mediated overexpression of long non-coding RNA GAS5 reduces invasion by mediating MMP2 expression and activity in human melanoma cells. Int J Oncol 2016;48:1509-1518.

-20 Cai H, Xue Y, Wang P, Wang Z, Li Z, Hu Y, Li Z, Shang X, Liu Y: The long noncoding RNA TUG1 regulates bloodtumor barrier permeability by targeting miR-144. Oncotarget 2015;6:19759-19779.

21 Cheetham SW, Gruhl F, Mattick JS, Dinger ME: Long noncoding RNAs and the genetics of cancer. Br J Cancer 2013;108:2419-2425.

-22 Modali SD, Parekh VI, Kebebew E, Agarwal SK: Epigenetic regulation of the lncRNA MEG3 and its target c-MET in pancreatic neuroendocrine tumors. Mol Endocrinol 2015;29:224-237.

23 Galiveti CR, Raabe CA, Konthur Z, Rozhdestvensky TS: Differential regulation of non-protein coding RNAs from Prader-Willi Syndrome locus. Sci Rep 2014;4:6445.

24 Cuccuru G, Lanzi C, Cassinelli G, Pratesi G, Tortoreto M, Petrangolini G, Seregni E, Martinetti A, Laccabue D, Zanchi C, Zunino F: Cellular effects and antitumor activity of RET inhibitor RPI-1 on MEN2A-associated medullary thyroid carcinoma. J Natl Cancer Inst 2004;96:1006-1014. 